\title{
Rapid diagnosis of bacterial meningitis by the detection of a fatty acid marker in CSF with gas chromatography- mass spectrometry and selected ion monitoring
}

\author{
G. L. FRENCH, C. Y. CHAN, D. POON, S. W. CHEUNG and A. F. B. CHENG
}

Department of Microbiology, The Chinese University of Hong Kong and the Prince of Wales Hospital, Shatin, New Territories, Hong Kong

\begin{abstract}
Summary. A chemical marker of bacterial meningitis was sought by comparing derivatives of sterile cerebrospinal fluid (CSF) with cultures of organisms in spinal fluid and artificial media. The technique of gas chromatography-mass spectrometry with selected ion monitoring (GC-MS-SIM) was used, optimised for the analysis of fatty acids. Twenty candidate ions were screened, and an ion of mass: charge ratio $(\mathrm{m} / \mathrm{e}) 268$ was chosen for detection in clinical specimens. The origin of this marker is unknown, but it is probably the molecular ion of a C16:1 fatty acid. In 135 clinical specimens of CSF examined, the $\mathrm{m} / \mathrm{e} 268$ ion was found to be a useful marker for the common organisms that cause bacterial meningitis, giving a sensitivity of $88 \%$ and a specificity of $98 \%$. The method was more rapid and more sensitive than conventional microscopy and culture, but CSF containing coagulase-negative staphylococci, Mycobacterium tuberculosis, Cryptococcus neoformans and some other uncommon pathogens gave inconsistent results. Many organisms produced characteristic ion profiles with multiple-ion monitoring, and this method of chemical analysis holds promise for the rapid diagnosis of bacterial infections to genus or species level.
\end{abstract}

\section{Introduction}

Various laboratory investigations of cerebrospinal fluid (CSF) have been proposed for the rapid diagnosis of bacterial meningitis. These include assay of CSF sugars ${ }^{1}$ and lactate ${ }^{2-7}$ the detection of bacterial antigens by immunological methods, ${ }^{8}$ and the detection of endotoxin. ${ }^{9}$ However, none of these tests is entirely satisfactory. Sugar and lactate concentrations may be altered in conditions other than bacterial meningitis; ${ }^{6,7}$ antigen detection is more sensitive than conventional Gram's staining and bacterial culture, but reagents are available for only a limited number of causative organisms; and the detection of endotoxin, while specific and rapid, is applicable only to infections with gram-negative organisms.

Chemical analysis by gas-liquid chromatography has been used for the rapid detection of bacterial metabolic products or cell constituents in CSF. ${ }^{10-15}$ We have previously shown that tuberculostearic acid (TBSA), a characteristic component of mycobacteria, is a specific marker of mycobacterial infection and may be detected rapidly and with high sensitivity in sputum ${ }^{16}$ and

Received 22 Feb. 1989; accepted 24 May 1989.
$\mathrm{CSF}^{17}$ by the technique of gas chromatographymass spectrometry with selected ion monitoring (GC-MS-SIM). In the present study, we have used GC-MS-SIM to screen CSF for fragment ions of other fatty acids that might be chemical markers of meningitis caused by other bacterial species.

\section{Materials and methods \\ Organisms and culture conditions}

Fifty strains representing 22 species of bacteria (including mycobacteria) and fungi were used for preliminary experimental studies (table I). These were mainly recent clinical isolates and included the common organisms of bacterial and fungal meningitis. Mycobacterium tuberculosis was cultured on Lowenstein-Jensen medium and incubated for 6-8 weeks at $37^{\circ} \mathrm{C}$. All other organisms were grown on chocolate blood agar, in Brain Heart Infusion Broth, and in sterile pooled CSF with and without supplementary glucose $(0.1 \%, w / v)$, and were incubated in air with $\mathrm{CO}_{2} 5 \%$ at $37^{\circ} \mathrm{C}$ for $18-24 \mathrm{~h}$.

\section{Standards and reagents}

Standard methyl esters of fatty acids were purchased from Sigma (St Louis, MO, USA). Sodium hydroxide 
Table I. Detection of ion m/e 268 in in-vitro cultures

\begin{tabular}{lc}
\hline Organisms & $\begin{array}{c}\text { Number positive/number } \\
\text { tested } \\
\text { for } \mathrm{m} / \mathrm{e} 268\end{array}$ \\
\hline Gram-negative & $16 / 17$ \\
Haemophilus influenzae type b & $2 / 2$ \\
Neisseria meningitidis & $1 / 1$ \\
Escherichia coli & $2 / 2$ \\
Klebsiella pneumoniae & $1 / 1$ \\
Enterobacter cloacae & $1 / 1$ \\
Citrobacter freundii & $1 / 1$ \\
Serratia liquefaciens & $1 / 1$ \\
Salmonella spp. & $2 / 3$ \\
Acinetobacter anitratus & $1 / 1$ \\
Flavobacterium meningosepticum & $1 / 1$ \\
Pseudomonas aeruginosa & $2 / 2$ \\
Pseudomonas cepacia & $1 / 1$ \\
Gram-positive & $16 / 23$ \\
Streptococcus pneumoniae & $2 / 2$ \\
Streptococcus agalactiae & $2 / 2$ \\
Streptococcus suis & $2 / 3$ \\
Staphylococcus aureus & $4 / 5$ \\
Coagulase-negative staphylococci & $5 / 10$ \\
Listeria monocytogenes & $1 / 1$ \\
Mycobacteria & $1 / 1$ \\
Mycobacterium tuberculosis & $1 / 1$ \\
Fungi & $2 / 4$ \\
Crytococcus neoformans & $1 / 3$ \\
Candida albicans & $1 / 1$ \\
\hline & \\
\hline
\end{tabular}

(Sigma), methanol (BDH Chemicals, Poole), chloroform and n-hexane (Reidel de Haan, Hannover, Germany), boron trichloride $10 \%$ in methanol (Sigma) and hydrochloric acid were all of reagent grade and were used without further purification.

\section{Preparation of specimens for GC-MS}

Cultures on solid media were transferred with a sterile loop to $1 \mathrm{ml}$ of sterile distilled water in reaction vials. Broth and CSF cultures were centrifuged at $3000 \mathrm{~g}$ for 15 min, and the resultant pellets washed twice with phosphate-buffered saline and then resuspended in $1 \mathrm{ml}$ of distilled water in reaction vials. Control samples of uninoculated media, sterile pooled CSF with and without glucose, human blood, concentrated human leucocytes and serial dilutions of bacterial cells in brain-heart infusion broth were also analysed. Samples in reaction vials were saponified with $2 \mathrm{ml}$ of sodium hydroxide $5 \%$ $\mathrm{w} / \mathrm{v}$ in methanol-water $(1: 1, \mathrm{v}: \mathrm{v})$, heated at $100^{\circ} \mathrm{C}$ for 30 min, cooled, and then acidified to $\mathrm{pH} 2.0$ with $2 \mathrm{ml}$ of $6 \mathrm{~N}$ $\mathrm{HCl}$. Fatty acids were extracted with $4 \mathrm{ml}$ of chloroform:hexane $(1: 4, v: v)$, dried under nitrogen, and methylated with $4 \mathrm{ml}$ of boron trichloride $10 \%$ in methanol at $100^{\circ} \mathrm{C}$ for $30 \mathrm{~min}$. Methylated fatty acid esters were extracted a second time with chloro- form:hexane, and $5 \mu \mathrm{l}$ of the resultant extracts were injected into the gas chromatograph for analysis. Methylated samples could be stored at $-20^{\circ} \mathrm{C}$ until injection without chemical changes.

\section{Gas chromatography-mass spectrometry}

The GC-MS equipment was manufactured by HewlettPackard, Avondale, USA, and comprised an HP 5970A quadrupole mass selective detector coupled to an HP $5880 \mathrm{~A}$ gas chromatograph and controlled by an HP 9825B desk-top computer. The chromatographic column was a $25 \mathrm{~m}$ High Performance Capillary Column of crosslinked methylsilicone (OV-1) with an internal diameter of $0.2 \mathrm{~mm}$ and film thickness of $0.33 \mu \mathrm{m}$ (HewlettPackard). Helium was used as the carrier gas. The injector port and interface temperature were set at $260^{\circ} \mathrm{C}$. The oven temperature started at $110^{\circ} \mathrm{C}$ with an initial run time of $3 \mathrm{~min}$, and then increased at a rate of $6^{\circ} \mathrm{C} /$ min to a final temperature of $260^{\circ} \mathrm{C}$ which was maintained for $10 \mathrm{~min}$. The mass selective detector was operated under a vacuum of $1.5 \times 10^{-5}$ torr, and at an analyser temperature of $200^{\circ} \mathrm{C}$.

Initial screening of potential chemical markers in invitro cultures was performed by selected monitoring of ions at the mass: charge $(\mathrm{m} / \mathrm{e})$ ratios of $240,242,254,256$, 
$258,268,270,282,284,286,294,296,298,310,312,314$, $324,326,340$, and 354 . These are the $\mathrm{m} / \mathrm{e}$ values of molecular ions of C14-C22 fatty acid methyl esters. The ion of $\mathrm{m} / \mathrm{e} 268$ (probably the molecular ion of a C16:1 fatty acid methyl ester) was later chosen as the marker for the diagnosis of bacterial meningitis, and m/e 242 was selected as a retention-time standard. Clinical specimens were then analysed by monitoring these two ions only.

\section{Clinical specimens of CSF}

We analysed 135 clinical specimens of CSF that had been submitted to the diagnostic laboratory for microbiological investigation, and reviewed the patients' clinical records retrospectively. Clinical meningitis was judged by signs and symptoms of meningism, fever, peripheral leucocytosis, typical CSF changes, microbiological findings and clinical response to appropriate therapy. Specimens were then divided into the following seven groups (table II).

Group $A$ (Proven bacterial meningitis): 22 purulent specimens that yielded bacteria on culture (18 specimens) or in which specific bacterial antigens were detected (four specimens). The organisms detected were Haemophilus influenzae (7), Streptococcus pneumoniae (5), Escherichia coli (3), Salmonella spp. (2), Str. suis (2), Str. agalactiae (2) and Klebsiella sp. (1). In nine of the specimens in this group no organisms were seen on microscopy.

Group B (Probable meningitis): two specimens with no detectable bacteria from patients who were nevertheless judged to have had bacterial meningitis on the basis of their CSF changes, clinical symptoms and response to therapy.

Group $C$ (Cryptococcal meningitis): five specimens that yielded Cryptococcus neoformans; yeasts were seen in India-ink preparations of two of these.

Group $D$ (Tuberculous meningitis): five microscopynegative specimens that contained TBSA but failed to yield mycobacteria. They were obtained from patients with typical clinical pictures of tuberculous meningitis.

Group $E$ (Abacterial meningitis): 28 sterile specimens of CSF with abnormally raised white cell counts but no organisms seen on microscopy. The patients were judged not to have bacterial meningitis. The majority of these specimens had a lymphocytic cellular reaction with normal CSF glucose concentrations. However, two specimens showed a polymorph leucocytosis and CSF glucose depletion--these were from a single patient with rickettsial meningitis. ${ }^{18}$

Group F (Control sterile CSF): 62 specimens in which there was no elevation of white cell count and no organisms; they were obtained from patients without meningitis.

Group $G$ (Contaminated CSF): 11 specimens containing few white cells, that showed scanty gram-positive organisms on microscopy or yielded coagulase-negative staphylococci on culture. These were from patients who were judged retrospectively not to have had bacterial meningitis.
Each CSF specimen was subjected to microscopy, culture and biochemical analysis by standard procedures. In appropriate specimens the antigens of $H$. influenzae, Str. pneumoniae and Neisseria meningitidis were sought by commercial antigen-detection kits (Wellcogen, Wellcome Diagnostics; Slidex, Biomerieux, Phadebact, Pharmacia Diagnostics).

The remaining material $(10 \mu \mathrm{l}-0.5 \mathrm{ml})$ was stored at $-20^{\circ} \mathrm{C}$ before GC-MS analysis.

\section{Results}

Derivatised pure bacterial cultures in artificial media and sterile CSF were analysed by GC-MSSIM for the molecular ions of methyl esters of common bacterial fatty acids. Analysis of these preliminary results suggested that an ion of $\mathrm{m} / \mathrm{e} 268$ with a retention time of $22.60 \pm 0.2 \mathrm{~min}$ was a marker for the presence of bacteria and yeasts. This ion, which is probably the molecular ion of a C16:1 fatty acid methyl ester, was consistently detected in extracts of all organism cultures except for those of Cryptococcus neoformans, coagulase-negative staphylococci, Staphylococcus aureus, Str. suis and Salmonella spp., which gave variable results (table I). The detection limit for this marker was approximately five bacterial cells when determined by the viable plate count method. The ion was not detected in extracts of sterile CSF, culture media or human leucocytes (figure), and was, therefore, selected as a potential chemical marker for bacterial meningitis. Ions of $\mathrm{m} / \mathrm{e} 242$ (probably fragments of long chain fatty acids, including the molecular ion of a C14 fatty acid methyl ester), were detected in all specimens of CSF. These eluted with relative retention times of 19.4 and $23.2 \mathrm{~min}$, and were used as internal controls for retention times (figure.)

Ion m/e 268 was detected in CSF from 21 of 22 patients with proven bacterial meningitis (including the four that were culture-negative but antigenpositive), but was not detected in the two specimens in Group B designated probable bacterial meningitis. The ion was detected in only one of 62 sterile control CSF specimens and in only one (from a patient with herpes encephalitis) of 28 specimens from abacterial meningitis (table II). The sensitivity of the method was thus 21 out of $24(87.5 \%)$ and the specificity 88 out of $90(97.8 \%)$.

The ion was found in three out of five specimens from patients with cryptococcal meningitis and in three out of five specimens from patients with tuberculous meningitis (table II). It was also detected in three of 11 contaminated specimens of CSF that yielded coagulase-negative staphylococci (table II). 


\section{Discussion}

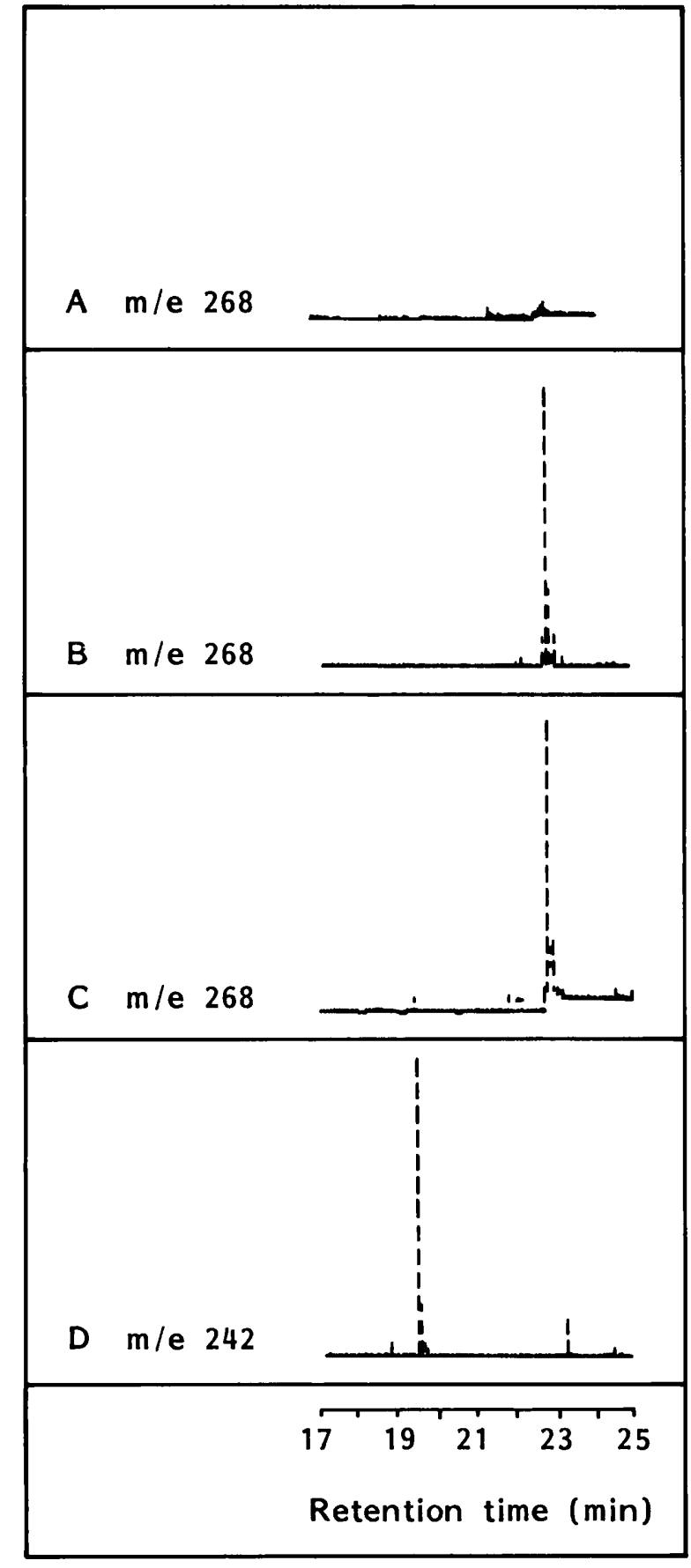

Figure. GC-MS-SIM of derivatised extracts of CSF. (A), sterile control CSF showing absence of ion $\mathrm{m} / \mathrm{e} 268 ;(B)$, an in-vitro culture of $H$. influenzae in pooled sterile CSF, showing a large peak of ion $\mathrm{m} / \mathrm{e} 268$ eluting at a relative retention time of 22.6 min; (C), a clinical specimen of CSF infected with $H$. influenzae, showing a similar peak for ion $\mathrm{m} / \mathrm{e} 268$; (D), control sterile CSF monitored for ions of $\mathrm{m} / \mathrm{e} 242$ which are used as retention time controls.
The use of gas chromatography for the detection of chemical markers of infection has been investigated by several workers. Amundson et al. ${ }^{1}$ analysed carbohydrates in CSF and found that specimens from patients with meningitis had altered concentrations of various sugars, but they examined few clinical specimens. Some workers have found a raised CSF lactic acid concentration to be strongly associated with bacterial meningitis, ${ }^{2-5}$ but others believe this marker to be unreliable. ${ }^{6,7}$ Others have pursued a different strategy: organisms were grown in artificial culture, characteristic bacterial products were defined, and these products identified in clinical specimens of various body fluids. Mitruka and co-workers ${ }^{19,20}$ demonstrated characteristic chromatographic patterns from different bacterial cultures and from sera of infected patients, but did not identify the component compounds. Brooks and colleagues ${ }^{10-13}$ identified chromatographic 'fingerprints' as well as specific bacterial products in several types of clinical specimen, including CSF. LaForce and colleagues ${ }^{14,15}$ examined fatty acid and carbohydrate profiles of the common bacterial agents of meningitis and found these to be useful for the diagnosis of meningitis in children and experimental infections in dogs. However, most of these techniques have been applied to only a small number of clinical specimens, and none has become established for routine use.

Another technique for the detection of trace amounts of specific bacterial compounds is gas chromatography-mass spectrometry combined with selected ion monitoring. General markers of bacterial infection such as $\beta$-hydroxymyristic acid ${ }^{21}$ and muramic acid ${ }^{22}$ have been identified by this method, as well as more specific ones such as tuberculostearic acid, the presence of which is indicative of mycobacterial infection ${ }^{16,17}$ and Darabinitol, associated with invasive candidiasis. $^{23,24}$

In the present study we used GC-MS-SIM to identify potential chemical markers of bacterial meningitis. The chemical composition of methylated derivatives of sterile CSF and in-vitro cultures of various bacteria and yeasts were compared. Specimens were analysed by GC-MS with simultaneous monitoring of 20 selected ions associated with common bacterial fatty acids. There were numerous differences between the ion patterns of sterile and artificially infected CSF. It is possible that with sufficient numbers of specimens and the use of multivariate statistical techniques, multiple 
Table II. Detection of ion $\mathrm{m} / \mathrm{e} 268$ in CSF of patients with meningitis and controls

\begin{tabular}{|c|c|c|c|c|c|c|c|}
\hline \multirow[b]{2}{*}{ CSF specimen group } & \multirow{2}{*}{$\begin{array}{l}\text { Number } \\
\text { tested }\end{array}$} & \multicolumn{2}{|c|}{ Microscopy } & \multicolumn{2}{|c|}{ Culture } & \multicolumn{2}{|c|}{ Ion $\mathrm{m} / \mathrm{e} 268$} \\
\hline & & + & - & + & - & + & - \\
\hline A: Proven bacterial meningitis & 22 & 13 & 9 & 18 & $4^{*}$ & 21 & 1 \\
\hline B: Probable bacterial meningitis & 2 & 0 & 2 & 0 & 2 & 0 & 2 \\
\hline C: Cryptococcal meningitis & 5 & 2 & 3 & 5 & 0 & 3 & 2 \\
\hline D: Tuberculous meningitis & 5 & 0 & 5 & 1 & 4 & 3 & 2 \\
\hline E: Abacterial meningitis & 28 & 0 & 28 & 0 & 28 & $1 \dagger$ & 27 \\
\hline F : Control sterile CSF & 62 & 0 & 62 & 0 & 62 & 1 & 61 \\
\hline G: Contaminated CSF & 11 & 6 & 5 & 7 & 4 & 3 & 8 \\
\hline
\end{tabular}

* Culture-negative but antigen-positive by latex agglutination.

$\dagger$ Herpes encephalitis.

ion groups could be selected as markers of specific bacterial species. However, in this preliminary study, a single ion was chosen for differentiation of infected and non-infected specimens by visual inspection.

Ion $\mathrm{m} / \mathrm{e} 268$, with a relative retention time of $22 \cdot 6 \pm 0.2$ min was chosen as the marker of bacterial meningitis. This ion was probably a methyl ester of a C16:1 fatty acid, but its exact identity was not determined. Ion $\mathrm{m} / \mathrm{e} 268$ was detected in the CSF of 21 out of 22 cases of proven bacterial meningitis. Salmonella group B was isolated from the single negative specimen, and this correlated with the finding that pure cultures of Salmonella spp. did not always produce this marker. Three culture-negative specimens of CSF from patients with clinical meningitis produced ion $\mathrm{m} / \mathrm{e} 268$, and these were later shown by latex agglutination to be positive for $H$. influenzae type b (2 cases) and Str. agalactiae antigens. Thus, the detection of ion $\mathrm{m} / \mathrm{e} 268$ was a more sensitive method for the diagnosis of bacterial meningitis than conventional microscopy and culture. However, the ion was not detected in two microscopy-, culture- and antigen-negative specimens from patients thought clinically to have had bacterial meningitis. These two specimens must presumably have contained very little bacterial material which could not be detected by any of the methods employed. The sensitivity of this marker for the detection of bacterial meningitis was thus $88 \%$. Ion $\mathrm{m} / \mathrm{e} 268$ was detected in only one of 62 control specimens of sterile CSF from patients without clinical meningitis, and in one of 28 specimens from patients with abacterial meningitis. These figures give a specificity of $98 \%$.

Ion $\mathrm{m} / \mathrm{e} 268$ was detected inconsistently in extracts of pure cultures of coagulase-negative staphylococci, cryptococci and mycobacteria, and it was also found to be unreliable as a marker of these organisms in clinical specimens. Contaminated CSF from eleven patients without clinical evidence of meningitis yielded coagulase-negative staphylococci, and ion m/e 268 was detected in three of these. Ion $\mathrm{m} / \mathrm{e} 268$ is thus unhelpful in the differentiation between meningitis and contamination with coagulase-negative staphylococci. The ion was found in only three out of five smearnegative, culture-negative cases of tuberculous meningitis diagnosed by the detection of TBSA, and in three out of five culture-positive specimens from cases of cryptococcal meningitis. Although we did not test in-vitro cultures of rickettsia, two CSF specimens from a patient with presumptive rickettsial meningitis also gave negative results for ion $\mathrm{m} / \mathrm{e} 268$.

The detection of chemical fragment ions in CSF is thus a potentially useful tool for the rapid diagnosis of bacterial meningitis. The detection of ion $\mathrm{m} / \mathrm{e} 268$ is more sensitive than conventional microscopy and gives a result within $2 \mathrm{~h}$. With the limited number of clinical specimens we were able to test, this ion appears to be a good marker for many of the common pathogens of bacterial meningitis (although we tested no CSF from cases of meningococcal meningitis), but gives variable results for infection with mycobacteria, cryptococci, staphylococci and salmonellae. The sensitivity and specificity of this method could probably be improved by the simultaneous analysis of additional ions.

This study demonstrates that the screening of body fluids for fragment ions of bacterial components can be used to search for chemical markers of infection. The ion patterns of individual orga- 
nisms can be reproduced in infected clinical specimens, and this methodology has potential for rapid bacterial diagnosis to the genus or species level. However, it is unlikely that mass spectrometry

\section{REFERENCES}

1. Amundson S, Braude A I, Davis C E. Rapid diagnosis of infection by gas-liquid chromatography: analysis of sugars in normal and infected cerebrospinal fluid. Appl Microbiol 1974; 28 : 298-302.

2. Controni G, Rodriguez W J, Hicks J M et al. Cerebrospinal fluid lactic acid levels in meningitis. J Pediatr 1977; 91 : 379-384.

3. Ferguson I R, Tearle P V. Gas liquid chromatography in the rapid diagnosis of meningitis. J Clin Pathol 1977; 30: 1163-1167.

4. Brook I, Bricknell K S, Overturf G D, Finegold S M. Measurement of lactic acid in cerebrospinal fluid of patients with infections of the central nervous system. J Infect Dis 1978; 137: 384-390.

5. Komorowski R A, Farmer S G, Hanson G A, Hause L L. Cerebrospinal fluid lactic acid in diagnosis of meningitis. J Clin Microbiol 1978; 8: 89-92.

6. D'Souza E, Mandal B K, Hooper J, Parker L. Lactic acid concentration in cerebrospinal fluid and differential diagnosis of meningitis. Lancet 1978; 2: 579-580.

7. Lannigan R, MacDonald M A, Marrie T J, Haldane E V. Evaluation of cerebrospinal fluid lactic acid levels as an aid in differential diagnosis of bacterial and viral meningitis in adults. $J$ Clin Microbiol $1980 ; 11$ : 324327.

8. Greenwood B M, Whittle H C, Dominic-Rajkovic O. Counter-current immunoelectrophoresis in the diagnosis of meningococcal infections. Lancet 1971; 2: 519521.

9. Berman N S, Siegel S E, Nachum R, Lipsey A, Leedom J. Cerebrospinal fluid endotoxin concentrations in gramnegative bacterial meningitis. J Pediatr 1976; 88: 553556.

10. Schlossberg D, Brooks J B, Shulman J. Possibility of diagnosing meningitis by gas chromatography: cryptococcal meningitis. J Clin Microbiol 1976; 3: 239-245.

11. Craven R B, Brooks J B, Edman D C et al. Rapid diagnosis of lymphocytic meningitis by frequency-pulsed electron capture gas-liquid chromatography: differentiation of tuberculous, cryptococcal, and viral meningitis. $J$ Clin Microbiol 1977; 6: 27-32.

12. Brooks J B, Kellogg D S, Shepherd M E, Alley C C. Rapid differentiation of the major causative agents of bacterial meningitis by use of frequency-pulsed electron capture gas-liquid chromatography: analysis of acids. $J$ Clin Microbiol 1980; 11 : 45-51.

13. Brooks J B, Edman D C, Alley C C, Craven R B, Girgis will ever be widely available for routine diagnosis; therefore, chemical markers identified by GC-MSSIM will need to be detected by simpler methods in clinical practice.

N I. Frequency-pulsed electron capture gas-liquid chromatography and the tryptophan color test for rapid diagnosis of tuberculous and other forms of lymphocytic meningitis. J Clin Microbiol 1980; 12: 208-215.

14. Brice J L, Tornabene T G, LaForce F M. Diagnosis of bacterial meningitis by gas-liquid chromatography. $\mathbf{I}$. Chemotyping studies of Streptococcus pneumoniae, Haemophilus influenzae, Neisseria meningitidis, Staphylococcus aureus and Escherichia coli. J Infect Dis 1979; 140: 443-452.

15. LaForce F M, Brice J L, Tornabene $T$ G. Diagnosis of bacterial meningitis by gas-liquid chromatography. II. Analysis of spinal fluid. $J$ Infect Dis 1979; 140: 453464.

16. French G L, Chan C Y, Cheung S W, Oo K T. Diagnosis of pulmonary tuberculosis by detection of tuberculostearic acid in sputum by using gas chromatography-mass spectrometry with selected ion monitoring. J Infect Dis 1987 ; 156: 356-362.

17. French G L, Teoh R, Chan C Y, Humphries M J, Cheung $\mathrm{S}$ W, O'Mahony G. Diagnosis of tuberculous meningitis by detection of tuberculostearic acid in cerebrospinal fluid. Lancet $1987 ; 2$ : 117-119.

18. Woo M L, Leung J W C, French G L. Rickettsial infection presenting as culture-negative meningitis. Postgrad Med J 1988; 64: 614-616.

19. Mitruka B M, Kundargi R S, Jonas A M. Gas chromatography for rapid differentiation of bacterial infections in man. Med Res Eng 1972; 11 : 7-11.

20. Mitruka B M. Rapid automated identification of microorganisms in clinical specimens by gas chromatography. In: Heden C-G, Illeni T (eds) New approaches to the identification of microorganisms. New York, John Wiley and Sons. 1975: 123-154.

21. Maitra S K, Schotz M C, Yoshikawa T T, Guze L B. Determination of lipid $\mathrm{A}$ and endotoxin in serum by mass spectroscopy. Proc Natl Acad Sci USA 1978; 75: 3993-3997.

22. Fox A, Schwab J H, Cochran T. Muramic acid detection in mammalian tissues by gas-liquid chromatography-mass spectrometry. Infect Immun 1980; 29: 526-531.

23. Kiehn T E, Bernard E M, Gold J W M, Armstrong G. Candidiasis: detection by gas-liquid chromatography of D-arabinitol, a fungal metabolite, in human serum. Science 1979 ; 206 : 577-580.

24. Roboz J, Suzuki R, Holland J F. Quantification of arabinitol in serum by selected ion monitoring as a diagnostic technique in invasive candidiasis. $J$ Clin Microbiol $1980 ; 12:$ 594-601. 